\title{
\& Research Square \\ PLEK2 and SCN7A: novel biomarkers of non-small cell lung cancer
}

\section{Yongchang Liu}

Xiangya Hospital Central South University

Xi Li

Xiangya Hospital Central South University

\section{Ruimin Chang}

Xiangya Hospital Central South University

\section{Yufan Chen}

Xiangya Hospital Central South University

Yang Gao ( $\nabla$ dr.gao@csu.edu.cn )

Xiangya Hospital Central South University

\section{Research}

Keywords: differentially expressed genes, non-small cell lung cancer, bioinformatics, PLEK2, SCN7A, prognosis

Posted Date: March 31st, 2020

DOI: https://doi.org/10.21203/rs.3.rs-20024/v1

License: (c) (i) This work is licensed under a Creative Commons Attribution 4.0 International License. Read Full License 


\section{Abstract}

Background Lung cancer is the leading cause of cancer-related death globally, and non-small cell lung cancer (NSCLC) is the most common type of lung cancer. However, the diagnosis and prognosis of NSCLC remain dim. Our team has focused on identifying differentially expressed genes (DEGs) between NSCLC tissues and adjacent tissues, which may be useful as effective diagnostic markers that can better explain the progression of NSCLC.

Methods The Gene Expression Omnibus (GEO) database was used to screen the Gene Expression Omnibus series, which records the information of a large number of patients with primary NSCLC $(n>$ 50). Then, the DEGs were validated using Student's t -test. Gene Ontology (GO) and Kyoto Encyclopedia of Genes and Genomes (KEGG) pathway enrichment analyses were performed using DAVID. The prognosis information was analyzed separately using data obtained from three databases, Human Protein Atlas, Kaplan-Meier Plotter, and SurvExpress.

Results A series of 180 DEGs (33 upregulated and 147 downregulated genes), mainly involving genes associated with extracellular exosomes, focal adhesion, and cell adhesion, were identified via GO analysis. Subsequently, KEGG analysis demonstrated that focal adhesion, cell adhesion molecules, and PPAR signaling pathway were the most enriched pathways. Then, we paid particular attention to pleckstrin 2 (PLEK2) and sodium voltage-gated channel alpha subunit 7 (SCN7A), as they have not been investigated as cancer-related genes previously. Kaplan-Meier survival analysis illustrated that PLEK2 and SCN7A levels were significantly correlated with the prognosis of NSCLC.

Conclusions Our research found that, as potential biomarkers, both PLEK2 and SCN7A are related to the development and prognosis of NSCLC. They may be used in disease screening and prognosis. The clinical significance of these two genes deserves further investigation.

\section{Background}

Lung cancer, which includes non-small cell lung cancer (NSCLC) and small cell lung cancer, is the most deadly malignancy globally $[1,2]$. NSCLC accounts for $80-85 \%$ of all cases of lung carcinoma, and it includes three major histological types: lung adenocarcinoma, lung squamous cell carcinoma, and large cell carcinoma [3, 4]. Lung adenocarcinoma has surpassed lung squamous cell carcinoma as the most prevalent histologic subtype of lung carcinoma [5]. Currently, several treatments are available for NSCLC, such as surgery, chemotherapy, irradiation, and molecular targeted therapy [6]. However, the 5-year mortality rate is extremely poor for NSCLC, ranging from 51 to $99 \%$ depending on the TNM stage. Because of the metastatic nature of NSCLC and its untypical symptoms, patients are normally diagnosed with advance disease, and only $5 \%$ of patients with stage $\rrbracket$ experience long-term survival [7]. Most patients with stage IV NSCLC are ineligible for surgery, and their survival is consequently poor. Unfortunately, the symptoms of NSCLC are not sufficiently obvious to permit diagnosis at an early stage 
[8]. Therefore, new effective biomarkers for early diagnosis and prognosis would be of great value for improving the clinical outcomes of patients with NSCLC.

In the progression of NSCLC, some genes are overexpressed, whereas others are inhibited. For example, with the development of lung cancer, calreticulin (CANX) is significantly upregulated [9]. Therefore, the serum CANX level has been used as a biomarker for the early detection of lung cancer in the clinic. Moreover, biomarkers related to the diagnosis and prognosis of NSCLC could be used as new targets for molecular targeted therapies. Prior studies demonstrated the diagnostic value of growth factor receptor mutation. However, the use of bioinformatics in detecting and identifying biomarkers of NSCLC still needs to be actualized.

With the development of bioinformatics, genome-scale analysis has been widely used in the research of cancer, especially in identifying potential biomarkers related to the development and prognosis of cancer [10]. These identified biomarkers could be used to more precisely characterize patients and facilitate the development of personalized targeted therapy. Moreover, bioinformatics could help to reveal the characteristics of tumorigenesis, identify pathogenic genes, and improve our understanding of tumor biology and genetics [11]. To investigate new targets to improve the diagnosis rate of lung cancer and predict patient outcomes, we downloaded five transcriptome microarray datasets from the Gene Expression Omnibus (GEO) database and identified differentially expressed genes (DEGs) between tumor and adjacent samples. We also integrated the results of these datasets to identify the common DEGs and performed Gene Ontology (GO) and Kyoto Encyclopedia of Genes and Genomes (KEGG) analyses of these common DEGs to identify their biological roles and pathways. Additionally, we downloaded the prognostic information of the DEGs from three different platforms (Human Protein Atlas, Kaplan-Meier Plotter, and SurvExpress) and analyzed the results of these three platforms. We found that pleckstrin 2 (PLEK2) upregulation was associated with a poor prognosis. However, patients with lower sodium voltage-gated channel alpha subunit 7 (SCN7A) expression had worse overall survival rates. Our team aimed to determine the relationship between these two genes and the diagnosis and prognosis of NSCLC.

\section{Materials And Methods}

\section{NSCLC transcriptome microarray dataset}

Five NSCLC datasets (GSE19804, GSE27262, GSE43458, GSE75037, GSE103512), including 308 tumor samples and 207 normal lung samples, were downloaded from GEO (https://www.ncbi.nlm.nih.gov/geo). The inclusion criteria for these datasets were as follows: (1) primary NSCLC histology; (2) no history of radiotherapy or chemotherapy; and (3) more than 50 pathological specimens on each chip. The GSE19804, GSE23066, and GSE27262 datasets were generated from the same detecting microarray platform ([HG-U133_Plus_2] Affymetrix Human Genome U133 Plus 2.0 Array), whereas the GSE43458, GSE75037, and GSE103512 data sets were obtained from [HuGene-1_0-st] Affymetrix Human Gene 1.0 ST Array, Illumina HumanWG-6 v3.0 expression beadchip, and [HT_HG-U133_Plus_PM] Affymetrix HT HGU133+ PM Array Plate, respectively. The flowchart of our work is shown in Figure 1. 


\section{Functional enrichment analysis}

During functional enrichment analysis of the 180 common DEGs, DAVID (Database for Annotation, Visualization, and Integrated Discovery, https://david.ncifcrf.gov/) was utilized for GO and KEGG pathway analyses. Terms with $P<0.05$ were considered significantly enriched.

\section{Survival analysis}

TCGA NSCLC gene expression data and the associated clinical data were downloaded from three different platforms for survival analysis, namely the Human Protein Atlas (http://www.proteinatlas.org/), Kaplan-Meier Plotter (http://kmplot.com/analysis/index.php?p=background/), and SurvExpress (http://bioinformatica.mty.itesm.mx/). Further, we analyzed the results with significant differences from these three different databases.

\section{Statistical analysis}

R software (Vision 3.1.0) was used for statistical analysis. Bioinformatics and Evolutionary genomics (http://bioinformatics.psb.ugent.be/webtools/Venn/) and GraphPad Prism (Vision 5.02) were used to generate plots. Student's $t$-test was applied for comparisons between two groups. Survival analysis was performed using the Kaplan-Meier method, and the log-rank test was used to evaluate the statistical significance of the differences. Differences were considered statistically significant at $P<0.05$.

\section{Results}

\section{Identification of DEGs between lung adenocarcinoma and normal lung tissues}

The mRNA expression profiles of GSE19804 included 60 tumor samples and 60 normal samples (605 upregulated and 1389 downregulated genes). GSE103512 included 60 tumor samples and 9 normal samples (109 upregulated and 605 downregulated genes). GSE27262 included 25 tumor samples and 25 normal samples (1453 upregulated and 2272 downregulated genes). GSE43458 included 80 tumor samples and 30 normal samples (390 genes upregulated and 785 downregulated genes). GSE75037 included 83 tumor samples and 83 normal samples (1754 upregulated and 2252 downregulated genes). The DEGs was identified using R software. The cut-off criterion for the upregulated DEGs was $P<0.05$ and $\log$ fold-change $>1$. Similarly, the criterion for the downregulated DEGs was $P<0.05$ and log foldchange $<-1$. These criteria were previously validated $[11,12]$. Further two-dimensional hierarchical clustering revealed a marked difference of expression modules of the DEGs, with separate clusters between the normal and tumor tissues (Figure 2a-e). The common set of the five sets of DEGs included 180 elements (33 upregulated and 147 downregulated genes, Figure 3a-b). These 180 DEGs (Table 1) were common to all NSCLC samples analyzed, and they were considered relevant in the development and progression of this malignancy. PLEK2 was the highest ranked upregulated gene, in line with previous studies $[13,14]$. 
The cause of NSCLC is complex. To investigate the biological roles and mechanisms of this malignancy, our group performed GO (Figure 4) and KEGG analyses (Figure 5). In our research, most of the DEGs belonged to the biological process category, in which receptor internalization, angiogenesis, and the positive regulation of angiogenesis were the most related GO terms. Regarding cellular component, most terms were associated with extracellular exosomes and focal adhesion. Among the 10 enriched molecular function terms, seven were associated with molecular connections (Figure 4). Therefore, we inferred that the progression of NSCLC may be related to the dysregulation of cell adhesion. Additionally, the KEGG analysis revealed that focal adhesion, cell adhesion molecules, and PPAR signaling pathway were the top three enriched pathways (Figure 5), indicating that PPAR plays a critical role in NSCLC progression.

\section{The common part of the prognosis data of DEGs}

After actualizing the GO and KEGG analyses, we further inquired whether the elevated expression of DEGs in NSCLC could affect patient survival. We used prognosis data from the Human Protein Atlas, KaplanMeier Plotter, and SurvExpress. Then, we analyzed the results of each database with significant differences (Figure $6 a-b$ ), identifying 6 protective and 13 harmful factors. These results are presented in Table 2.

\section{PLEK2 and SCN7A dysregulation are indicators for prognosis of patients with NSCLC}

Patients with increased PLEK2 expression had a lower survival rate than those with reduced PLEK2 expression $(P<0.05$, Figure 7a). Similarly, lower SCN7A expression was linked to a poorer survival rate ( $P$ $<0.05$, Figure $7 b$ ). Interestingly, SLIT2, which had lower expression in tumor tissue than in normal tissue, also related with poorer outcomes (Figure 8).

\section{Discussion}

Aberrant expression of mRNA is widely considered a distinct characteristic of the development and progression of various cancers, especially lung cancer. To investigate the dysregulation of mRNA expression in NSCLC, our group integrated the results of five NSCLC transcriptome microarray datasets that are widely considered to have high credibility. Additionally, these five datasets were downloaded from different platforms, which allowed us to avoid the biases of the platforms. By integrating these five datasets, we largely ensured the credibility of our results. Our research suggests that PLEK2 and SCN7A may be used as potential biological markers for the early diagnosis of NSCLC.

Some of our DEGs were believed to be associated with lung adenocarcinoma in previous studies, such as TPX2 [15], IGF2BP3 [16], ANKRD22 [17], TMPRSS4 [18], SPP1 [19], MMP1 [20, 21], MMP12 [22], UBE2T [23], ECT2 [24, 25], SULF1 [26], HMGB3 [27], and CTHRC1 [28, 29]. Besides, we identified PLEK2 and SCN7A as potential biomarkers of NSCLC. PLEK2 was first cloned in 1999, and it is widely expressed in diverse adherent cell lines [30,31]. It is thus tempting to consider that PLEK2 is related to cytoskeletal rearrangement and cell spreading, as well as the development of large lamellipodia [32]. Moreover, 
lamellipodia are widely believed to participate in angiogenesis by endothelial cells and metastasis by melanoma cells [33-35]. Hamaguchi also reported that PLEK2 induced PI3-kinase-dependent F-actin reorganization and cell spreading [36]. Consistent with this hypothesis, our study revealed that PLEK2 may result in poor prognosis by enhancing the efficiency of tumor growth and the metastasis of NSCLC cells. SCN7A encodes a voltage-dependent sodium channel of the excitable membrane, and it is reported to be downregulated in colorectal carcinoma at the mRNA level [37]. However, the antitumor activity of SCN7A is unknown. Therefore, more elaborative research is needed.

Similarly, Liu reported that low elF3a expression is a negative factor in the development of colonic carcinoma $[38,39]$. In addition, KEGG pathway analysis indicated that the PPAR signaling pathway, cell adhesion molecules, and ECM-receptor interaction were the most significantly enriched pathways. Our results indicated some of these genes, which were found to play critical roles in the promotion of EMT and the invasion and migration of cells in previous studies [40, 41], were associated with the PPAR signaling pathway. Furthermore, the PPAR signaling pathway is related to cell adhesion and apoptosis [42], which is consistent with our results of GO analysis. PPAR, the isoforms of which include PPARa, PPAR $\beta$, and PPARy, belongs to the nuclear hormone receptor superfamily. PPARy controls tumor growth, cell proliferation, and cell invasiveness by inhibiting various signaling pathways such as the JAK/STAT, transforming growth factor (TGF), WNT/ $\beta$-catenin, and PI3K/ Akt pathways [43]. TGF is related to the occurrence and development of various cancer-related diseases [44, 45]. Furthermore, previous studies indicated that TGF promoted the malignancy, differentiation, and metastasis of tumors, such as NSCLC, pancreatic cancer, and colon cancer [46-49], by inducing EMT. Evidences have shown that the PPAR receptor was frequently overexpressed in lung cancer [50,51]. Recent studies [51, 52] indicated that TGF induces EMT through the PPAR pathway, leading to the differentiation, metastasis, and malignant transformation of lung cancer cells. In addition, PPARY induces MUC1-C ubiquitination and degradation, which are associated with various cancers such as colon, breast, ovarian, lung, and pancreatic cancers [53].Collectively, these observations indicate that PPAR signaling pathway may participate in NSCLC progression.

\section{Conclusion}

In this study, we examined the changes of gene expression in NSCLC and identified a series of DEGs, and we specifically identified potential roles of PLEK2 and SCN7A in the diagnosis and prognosis of NSCLC. They could also be new potential therapeutic targets that could improve the patient survival in NSCLC.

\section{Abbreviations}

NSCLC: non-small cell lung cancer; DEG: differentially expressed gene; GO: Gene Ontology; KEGG: Kyoto Encyclopedia of Genes and Genomes; PLEK2: pleckstrin 2; SCN7A: sodium voltage-gated channel alpha subunit 7

\section{Declarations}


Acknowledgements

Not applicable.

\section{Funding}

This work was supported by the Key Research and Development Program of Hunan Province (2016JC2039), Programs of Changsha Science and Technology Agent (kq1701080), Natural Science Foundation General Program of Hunan Province (2017JJ2386, 2018JJ3821), Clinic and Rehabilitation Research Foundation of Sinobioway group (xywm2015I37), National Natural Science Youth Foundation of China (81602027,81702928), Project of Scientific Research Plan of the Hunan Provincial Health Commission (C2019185), and Youth Foundation of Xiangya Hospital Central South University (2015Q01).

\section{Availability of data and materials}

The datasets used and/or analyzed during the current study are available from the corresponding author on reasonable request.

\section{Ethics approval and consent to participate}

Not applicable.

\section{Consent for publication}

Not applicable

\section{Competing interests}

The authors declare no competing financial interest.

\section{Authors' contributions}

Conception and design: Yang Gao, Xi Li, Yong-chang Liu

Critical revision for important intellectual content: Rui-min Chang, Yu-fan Chen, Yong-chang Liu

Final approval of the version to be published: Yang Gao, Xi Li, Rui-min Chang, Yong-chang Liu, Yu-fan Chen

Drafting of the article: Yang Gao, Yong-chang Liu

\section{References}

1. Peng W, Wang J, Shan B, Peng Z, Dong Y, Shi W, He D, Cheng Y, Zhao W, Zhang C, et al: Diagnostic and Prognostic Potential of Circulating Long Non-Coding RNAs in Non Small Cell Lung Cancer. Cell 
Physiol Biochem 2018, 49:816-827.

2. Mei Y, Liu YB, Hu DL, Zhou HH: Effect of RIF1 on response of non-small-cell lung cancer patients to platinum-based chemotherapy by regulating MYC signaling pathway. Int J Biol Sci 2018, 14:18591872.

3. Molina JR, Yang P, Cassivi SD, Schild SE, Adjei AA: Non-small cell lung cancer: epidemiology, risk factors, treatment, and survivorship. Mayo Clin Proc 2008, 83:584-594.

4. Huang T, Li J, Zhang C, Hong Q, Jiang D, Ye M, Duan S: Distinguishing Lung Adenocarcinoma from Lung Squamous Cell Carcinoma by Two Hypomethylated and Three Hypermethylated Genes: A Meta-Analysis. PLoS One 2016, 11:e0149088.

5. Davidson MR, Gazdar AF, Clarke BE: The pivotal role of pathology in the management of lung cancer. $J$ Thorac Dis 2013, 5 Suppl 5:S463-478.

6. Cho JH: Immunotherapy for Non-small-cell Lung Cancer: Current Status and Future Obstacles. Immune Netw 2017, 17:378-391.

7. Gulley JL, Spigel D, Kelly K, Chandler JC, Rajan A, Hassan R, Wong DJL, Leach J, Edenfield WJ, Wang D, et al: Avelumab (MSB0010718C), an anti-PD-L1 antibody, in advanced NSCLC patients: A phase $1 \mathrm{~b}$, open-label expansion trial in patients progressing after platinum-based chemotherapy. Journal Of Clinical Oncology 2015, 33.

8. Chalela R, Curull V, Enriquez C, Pijuan L, Bellosillo B, Gea J: Lung adenocarcinoma: from molecular basis to genome-guided therapy and immunotherapy. J Thorac Dis 2017, 9:2142-2158.

9. Kobayashi M, Nagashio R, Jiang SX, Saito K, Tsuchiya B, Ryuge S, Katono K, Nakashima H, Fukuda E, Goshima N: Calnexin is a novel sero-diagnostic marker for lung cancer. Lung Cancer 2015, 90:342345.

10. Gao YF, Mao XY, Zhu T, Mao CX, Liu ZX, Wang ZB, Li L, Li X, Yin JY, Zhang W, et al: COL3A1 and SNAP91: novel glioblastoma markers with diagnostic and prognostic value. Oncotarget 2016, 7:70494-70503.

11. Zhu T, Gao YF, Chen YX, Wang ZB, Yin JY, Mao XY, Li X, Zhang W, Zhou HH, Liu ZQ: Genome-scale analysis identifies GJB2 and ERO1LB as prognosis markers in patients with pancreatic cancer. Oncotarget 2017, 8:21281-21289.

12. Piao J, Sun J, Yang Y, Jin T, Chen L, Lin Z: Target gene screening and evaluation of prognostic values in non-small cell lung cancers by bioinformatics analysis. Gene 2018, 647:306-311.

13. Ooi AT, Gomperts BN: Molecular Pathways: Targeting Cellular Energy Metabolism in Cancer via Inhibition of SLC2A1 and LDHA. Clin Cancer Res 2015, 21:2440-2444.

14. Shiao SPK, Xiao H, Dong L, Wang X, Liu K, She J, Shi H: Genome wide DNA differential methylation regions in colorectal cancer patients in relation to blood related family members, obese and nonobese controls - a preliminary report. Oncotarget 2018, 9:25557-25571.

15. Orth M, Unger K, Schoetz U, Belka C, Lauber K: Taxane-mediated radiosensitization derives from chromosomal missegregation on tripolar mitotic spindles orchestrated by AURKA and TPX2. Oncogene 2018, 37:52-62. 
16. Zhao W, Lu D, Liu L, Cai J, Zhou Y, Yang Y, Zhang Y, Zhang J: Insulin-like growth factor 2 mRNA binding protein 3 (IGF2BP3) promotes lung tumorigenesis via attenuating p53 stability. Oncotarget 2017, 8:93672-93687.

17. Yin J, Fu W, Dai L, Jiang Z, Liao H, Chen W, Pan L, Zhao J: ANKRD22 promotes progression of nonsmall cell lung cancer through transcriptional up-regulation of E2F1. Scientific Reports 2017, 7.

18. Villalba M, Lopez L, Redrado M, Ruiz T, de Aberasturi AL, De IRN, Garcia D, Exposito F, De AC, AlvarezFernandez E: Development of biological tools to assess the role of TMPRSS4 and identification of novel tumor types with high expression of this prometastatic protein. Histology \& Histopathology 2017, 32:929-940.

19. Zhang H, Liu HB, Yuan DM, Wang ZF, Wang YF, Song Y: Prognostic value of secreted phosphoprotein-1 in pleural effusion associated with non-small cell lung cancer. BMC Cancer 2014, 14:280.

20. Hart K, Landvik NE, Lind H, Skaug V, Haugen A, Zienolddiny S: A combination of functional polymorphisms in the CASP8, MMP1, IL10 and SEPS1 genes affects risk of non-small cell lung cancer. Lung Cancer 2011, 71:123-129.

21. Hu J, Pan J, Luo ZG: MMP1 rs1799750 single nucleotide polymorphism and lung cancer risk: a meta-analysis. Asian Pac J Cancer Prev 2012, 13:5981-5984.

22. Wen $Y$, Cai L: [Research progress of matrix metalloproteinase 12 in non-small cell lung cancer]. Chinese Journal of Lung Cancer 2014, 17:30-33.

23. Perez-Pena J, Corrales-Sanchez V, Amir E, Pandiella A, Ocana A: Ubiquitin-conjugating enzyme E2T (UBE2T) and denticleless protein homolog (DTL) are linked to poor outcome in breast and lung cancers. Sci Rep 2017, 7:17530.

24. Justilien V, Jameison L, Der CJ, Rossman KL, Fields AP: Oncogenic Activity of Ect2 Is Regulated through Protein Kinase Cl-mediated Phosphorylation. Journal of Biological Chemistry 2011, 286:8149.

25. Murata Y, Minami Y, Iwakawa R, Yokota J, Usui S, Tsuta K, Shiraishi K, Sakashita S, Satomi K, lijima T: ECT2 amplification and overexpression as a new prognostic biomarker for early-stage lung adenocarcinoma. Cancer Science 2014, 105:490-497.

26. Heidari-Hamedani G, Vives RR, Seffouh A, Afratis NA, Oosterhof A, van Kuppevelt TH, Karamanos NK, Metintas M, Hjerpe A, Dobra K, Szatmari T: Syndecan-1 alters heparan sulfate composition and signaling pathways in malignant mesothelioma. Cell Signal 2015, 27:2054-2067.

27. Song N, Liu B, Wu JL, Zhang RF, Duan L, He WS, Zhang CM: Prognostic value of HMGB3 expression in patients with non-small cell lung cancer. Tumour Bio/ 2013, 34:2599-2603.

28. He W, Zhang H, Wang Y, Zhou Y, Luo Y, Cui Y, Jiang N, Jiang W, Wang H, Xu D: CTHRC1 induces nonsmall cell lung cancer (NSCLC) invasion through upregulating MMP-7/MMP-9. Bmc Cancer 2018, 18:400.

29. Wang Y, Lee M, Yu G, Hua L, Han X, Kim D: CTHRC1 activates pro-tumorigenic signaling pathways in hepatocellular carcinoma. Oncotarget 2017, 8:105238-105250. 
30. Parrish ML, Wright C, Rivers Y, Argilla D, Collins H, Leeson B, Loboda A, Nebozhyn M, Marton MJ, Lejnine S: cDNA targets improve whole blood gene expression profiling and enhance detection of pharmocodynamic biomarkers: a quantitative platform analysis. Journal of Translational Medicine,8,1(2010-09-25) 2010, 8:87.

31. Yuchun L, Steven R, Junichi F, Lisa S, Jay M, Edwards CK, Karl W, Kathleen S, Norris DA, Danute BD: Transcriptome profiling of whole blood cells identifies PLEK2 and C1QB in human melanoma. Plos One 2011, 6:e20971.

32. Roll RL, Bauman EM, Bennett JS, Abrams CS: Phosphorylated pleckstrin induces cell spreading via an integrin-dependent pathway. J Cell Biol 2000, 150:1461-1466.

33. Ridley A: Molecular switches in metastasis. Nature 2000, 406:466-467.

34. Clark EA, Golub TR, Lander ES, Hynes RO: Genomic analysis of metastasis reveals an essential role for RhoC. Nature 2000, 406:532-535.

35. Bittner M, Meltzer P, Chen Y, Jiang Y, Seftor E, Hendrix M, Radmacher M, Simon R, Yakhini Z, Ben-Dor A, et al: Molecular classification of cutaneous malignant melanoma by gene expression profiling. Nature 2000, 406:536-540.

36. Hamaguchi N, Ihara S, Ohdaira T, Nagano H, Iwamatsu A, Tachikawa H, Fukui Y: Pleckstrin-2 selectively interacts with phosphatidylinositol 3-kinase lipid products and regulates actin organization and cell spreading. Biochemical \& Biophysical Research Communications 2007, 361:270-275.

37. Beata O, Paweł O, Kamila DS, Katarzyna O, Piotr Z: Quantitative analysis of gene expression in fixed colorectal carcinoma samples as a method for biomarker validation. Molecular Medicine Reports 2016, 13:5084-5092.

38. Liu Z, Dong Z, Yang Z, Chen Q, Yi P, Yang Y, Ping C, Xin Z, Zhang JT: Role of elF3a (elF3 p170) in intestinal cell differentiation and its association with early development. Differentiation 2010, 75:652-661.

39. Zhu T, Gao Y, Li L, Wang L, Yin J, Zhou H, Zhang W, Liu Z: Aberration in translation initiation and associated diseases: Role of the eukaryotic translation initiation factor 3A. Zhong Nan Da Xue Xue Bao Yi Xue Ban 2017, 42:1204-1211.

40. Lin LC, Hsu SL, Wu CL, Hsueh CM: TGF $\beta$ can stimulate the $p(38) / \beta$-catenin/PPARy signaling pathway to promote the EMT, invasion and migration of non-small cell lung cancer ( 4460 cells). Clinical \& Experimental Metastasis 2014, 31:881-895.

41. Wu X, Zhang W, Hu Y, Yi X: Bioinformatics approach reveals systematic mechanism underlying lung adenocarcinoma. Tumori 2015, 101:281.

42. Zhang Z, Xu Y, Xu Q, Hou Y: PPARgamma against tumors by different signaling pathways. Onkologie 2013, 36:598-601.

43. Vallée A, Lecarpentier Y, Guillevin R, Vallée JN: Opposite Interplay Between the Canonical WNT/ $\boldsymbol{B}$ Catenin Pathway and PPAR Gamma: A Potential Therapeutic Target in Gliomas. Neuroscience Bulletin 2018:1-16. 
44. Chow G, Tauler J, Mulshine JL: Cytokines and growth factors stimulate hyaluronan production: role of hyaluronan in epithelial to mesenchymal-like transition in non-small cell lung cancer. J Biomed Biotechnol 2010, 2010:485468.

45. Kim ES, Kim MS, Moon A: Transforming growth factor (TGF)- $\beta$ in conjunction with H-ras activation promotes malignant progression of MCF10A breast epithelial cells. Cytokine 2005, 29:84-91.

46. Keshamouni VG, Reddy RC, Arenberg DA, Thannickal VJ, Kalemkerian GP, Standiford TJ: Peroxisome proliferator-activated receptor-gamma activation inhibits tumor progression in non-small-cell lung cancer. Oncogene 2004, 23:100-108.

47. Reka AK, Kurapati H, Narala VR, Bommer G, Chen J, Standiford TJ, Keshamouni VG: Peroxisome proliferator-activated receptor-gamma activation inhibits tumor metastasis by antagonizing Smad3mediated epithelial-mesenchymal transition. Molecular Cancer Therapeutics 2010, 9:3221.

48. Yoshizumi T, Ohta T, Ninomiya I, Terada I, Fushida S, Fujimura T, Nishimura G, Shimizu K, Yi S, Miwa $\mathrm{K}$ : Thiazolidinedione, a peroxisome proliferator-activated receptor-gamma ligand, inhibits growth and metastasis of HT-29 human colon cancer cells through differentiation-promoting effects. International Journal of Oncology 2004, 25:631-639.

49. Ohta T, Elnemr A, Yamamoto M, Ninomiya I, Fushida S, Nishimura G, Fujimura T, Kitagawa H, Kayahara M, Shimizu K: Thiazolidinedione, a peroxisome proliferator-activated receptor-gamma ligand, modulates the E-cadherin/beta-catenin system in a human pancreatic cancer cell line, BxPC3. International Journal of Oncology 2002, 21:37-42.

50. Giaginis C, Politi E, Alexandrou P, Sfiniadakis J, Kouraklis G, Theocharis S: Expression of peroxisome proliferator activated receptor-gamma (PPAR- $\gamma$ ) in human non-small cell lung carcinoma: correlation with clinicopathological parameters, proliferation and apoptosis related molecules and patients' survival. Pathology Oncology Research Por 2012, 18:875-883.

51. Theocharis S, Kanelli H, Politi E, Margeli A, Karkandaris C, Philippides T, Koutselinis A: Expression of peroxisome proliferator activated receptor-gamma in non-small cell lung carcinoma: correlation with histological type and grade. Lung Cancer 2002, 36:249.

52. Giaginis C, Politi E, Alexandrou P, Sfiniadakis J, Kouraklis G, Theocharis S: Expression of Peroxisome Proliferator Activated Receptor-Gamma (PPAR-y) in Human Non-small Cell Lung Carcinoma: Correlation with Clinicopathological Parameters, Proliferation and Apoptosis Related Molecules and Patients' Survival. Pathology \& Oncology Research 2012, 18:875-883.

53. Hou Y, Gao J, Xu H, Xu Y, Zhang Z, Xu Q, Zhang C: PPARgamma E3 ubiquitin ligase regulates MUC1-C oncoprotein stability. Oncogene 2014, 33:5619-5625.

\section{Tables}


Table 1: Common DEGs identified in NSCLC.

\begin{tabular}{ll}
\hline Expression & \multicolumn{1}{c}{ DEGs } \\
\hline TPX2,IGF2BP3,ANLN,CDK1,ANKRD22,TMPRSS4,SPP1,MMP1,MMP1 \\
2,UBE2T,SLC2A1,ECT2,FAM83A,CKMT1B,CEP55,CKMT1A,TFAP2A, \\
MKI67,SULF1,COL11A1,PLAU,HMGB3,DSP,SERINC2,BUB1,ASPM,P \\
LEK2,RRM2,TOP2A,COL10A1,CTHRC1,CDKN3,CENPF \\
\\
ABCA3,PEBP4,SUSD2,PAPSS2,FIGF,SLCO2A1,LAMP3,PTRF,SPTBN1, \\
LRRK2,CLEC3B,NDNF,FHL1,PHACTR1,KLF4,ZFP36,NDRG2,SVEP1, \\
TCF21,SFTPD,OLR1,GLIPR2,AGER,HOXA5,CD93,MSR1,TPPP3,FLI, \\
CLIC5,NPNT,CSRNP1,PDK4,SFTPA1,EFEMP1,SASH1,OGN,ESAM,CX \\
CL2,LYVE1,AKAP12,PDE5A,CPA3,ROBO4,ADIRF,HBB,RHOJ,SCN7A, \\
SLC39A8,SFTPC,TGFBR3,MYL9,LPL,CAT,LMCD1,CAV2,FOSB,MAR \\
CO,EPAS1,SLIT2,CYBRD1,C2orf40,AOX1,CYP4B1,MYADM,FAM216 \\
B,CHRDL1,DPYSL2,BTNL9,VEPH1,TIMP3,HBEGF,CRYAB,CD36,PTP \\
RB,AQP4,ITGA8,MT1M,IL1RL1,KLF6,EMP1,ABCA8,AOC3,DES,EDN \\
RB,SLIT3,MYH10,MYH11,DUSP1,SLPI,RGCC,VWF,ABI3BP,VSIG4,PL \\
EKHH2,C7,HEG1,IL6,ANGPT1,DUOX1,FERMT2,DPT,MFAP4,PECAM \\
1,IGSF10,SEPP1,SDPR,ID4,ADAMTS1,SORBS1,S1PR1,FOS,LIMCH1,L \\
DB2,CALCRL,CAV1,CLEC1A,JAM2,SPARCL1,INMT,METTL7A,ITM2 \\
A,AQP1,IL7R,FMO2,SOCS2,RBMS3,TGFBR2,PGC,ADH1B,NED9,FAB \\
P4,FCN3,PDLIM3,FAM107A,GPX3,TNXA,RAMP2,VIPR1,PLAC8,MA \\
MDC2,CLDN18,CDH5,CES1,PTGIS,FBLN5,HBA2,HBA1
\end{tabular}

Table 2: Common DEGs related with the prognosis of NSCLC.

\begin{tabular}{ll}
\hline & \multicolumn{1}{c}{ DEGs } \\
\hline Protective factors & SCN7A, CHRDL1, SLIT2, TCF21, CPA3, PHACTR1 \\
Harmful factors & CDKN3, PLEK2, RRM2, MMP12, IGF2BP3, CEP55, SPP1, SLC2A1, TOP2A, CENPF, ECT2, TPX2, ASPM \\
\hline
\end{tabular}

\section{Figures}




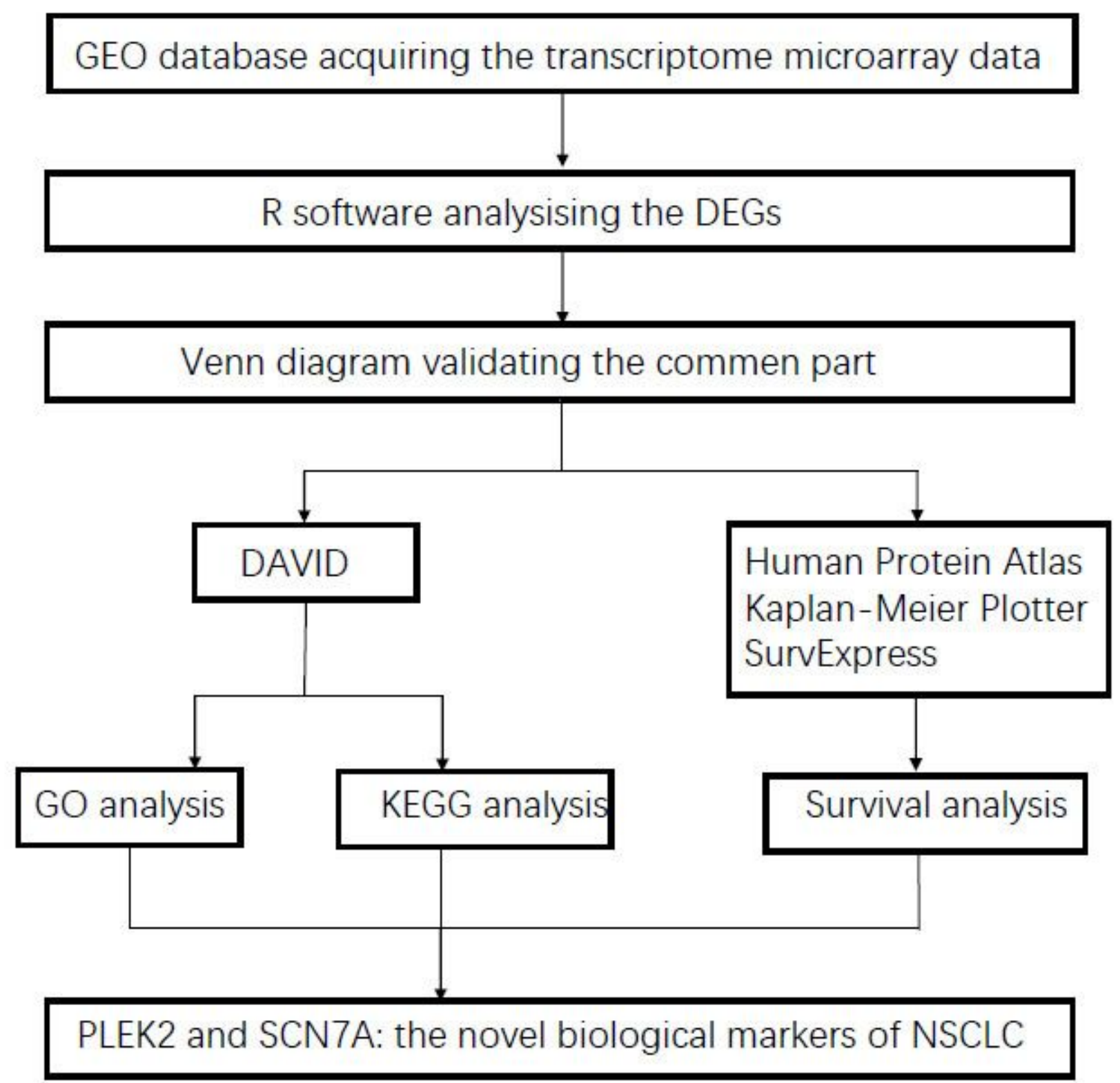

Figure 1

Workflow of the study. 

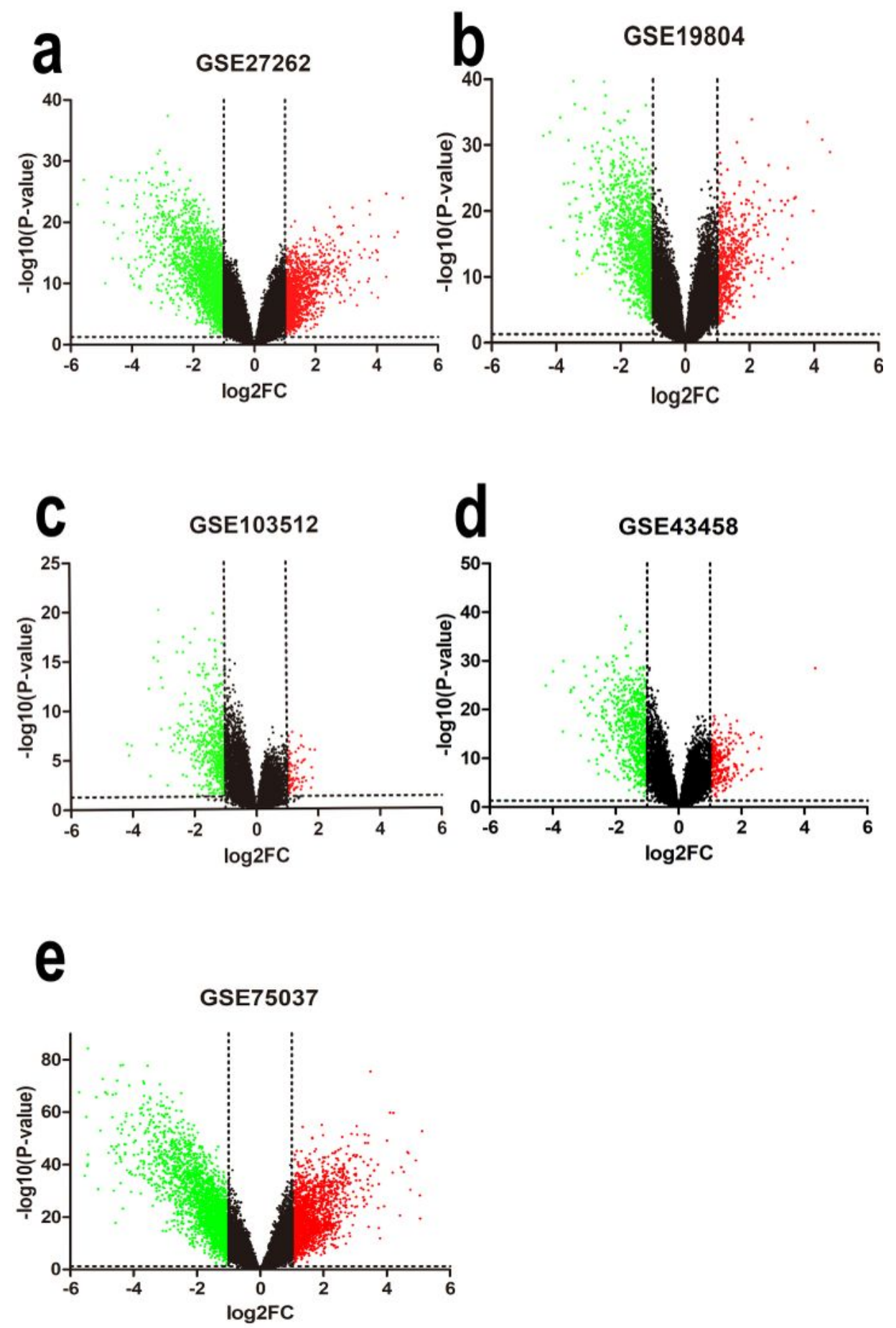

\section{Figure 2}

Identification of differentially expressed genes (DEGs) between non-small cell lung cancer (NSCLC) and adjacent tissues. (a-e) Volcano plots of DEGs. In GSE27262, 1453 genes were upregulated, and 2272 genes were downregulated (a). In GSE19804, 605 genes were upregulated, and 1389 genes were downregulated (b). In GSE103512, 109 genes were upregulated, and 605 genes downregulated (c). In 
GSE43458, 390 genes were upregulated, and 785 genes were downregulated (d). In GSE70537, 1754 genes were upregulated, and 2252 genes were downregulated (e).
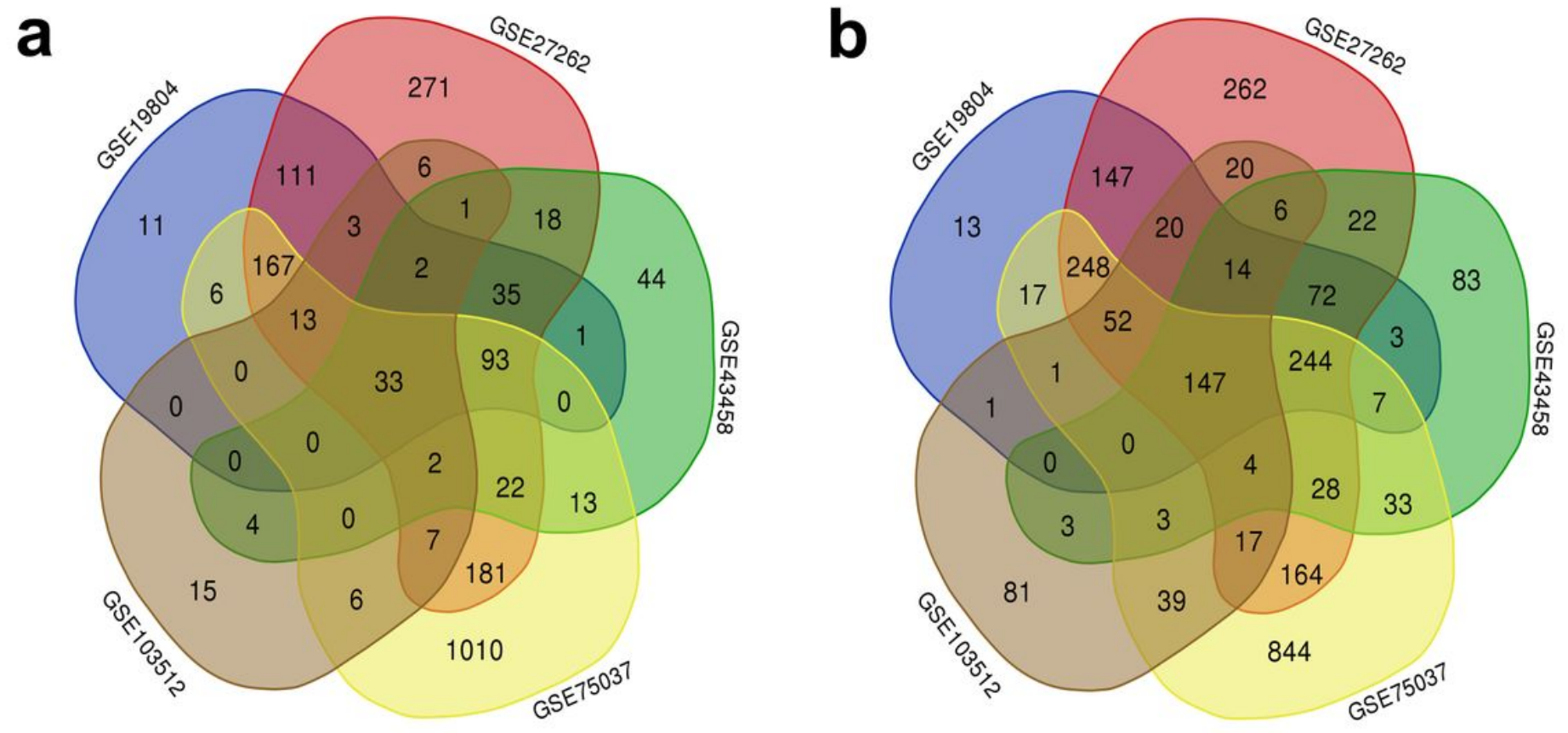

\section{Figure 3}

Venn diagram of the common genes of the five sets of differentially expressed genes, including both upand downregulated genes. There were 33 common upregulated genes (a) and 147 downregulated genes (b) in these five sets. 


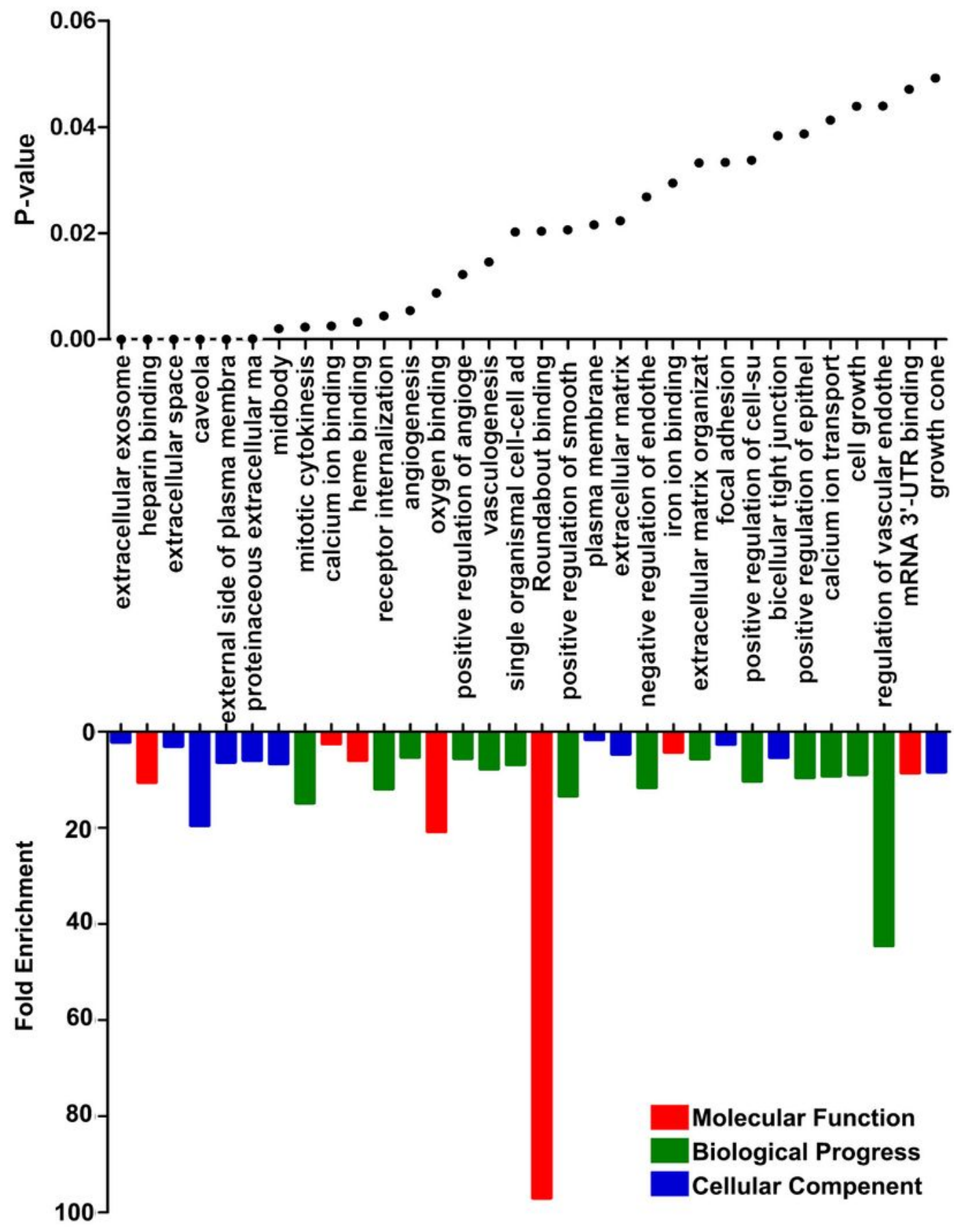

Figure 4

Gene Ontology (GO) analysis of the 180 common differentially expressed genes. The upper panel shows the $P$ value of each $\mathrm{GO}$ term, and the lower panel shows the enrichment of each GO term. 


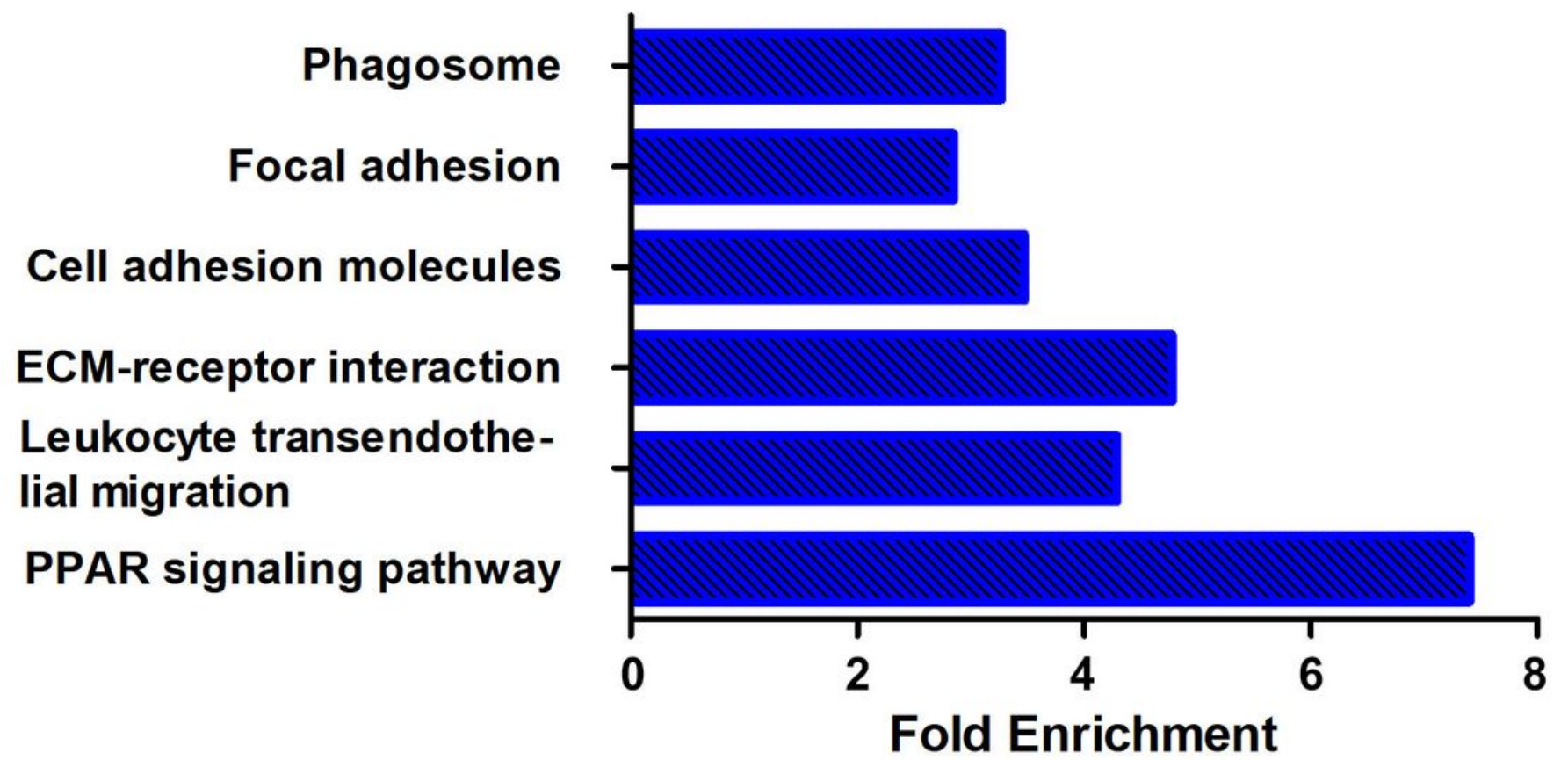

Figure 5

Kyoto Encyclopedia of Genes and Genomes pathway enrichment analysis of the 180 common differentially expressed genes significant at $\mathrm{P}<0.05$.
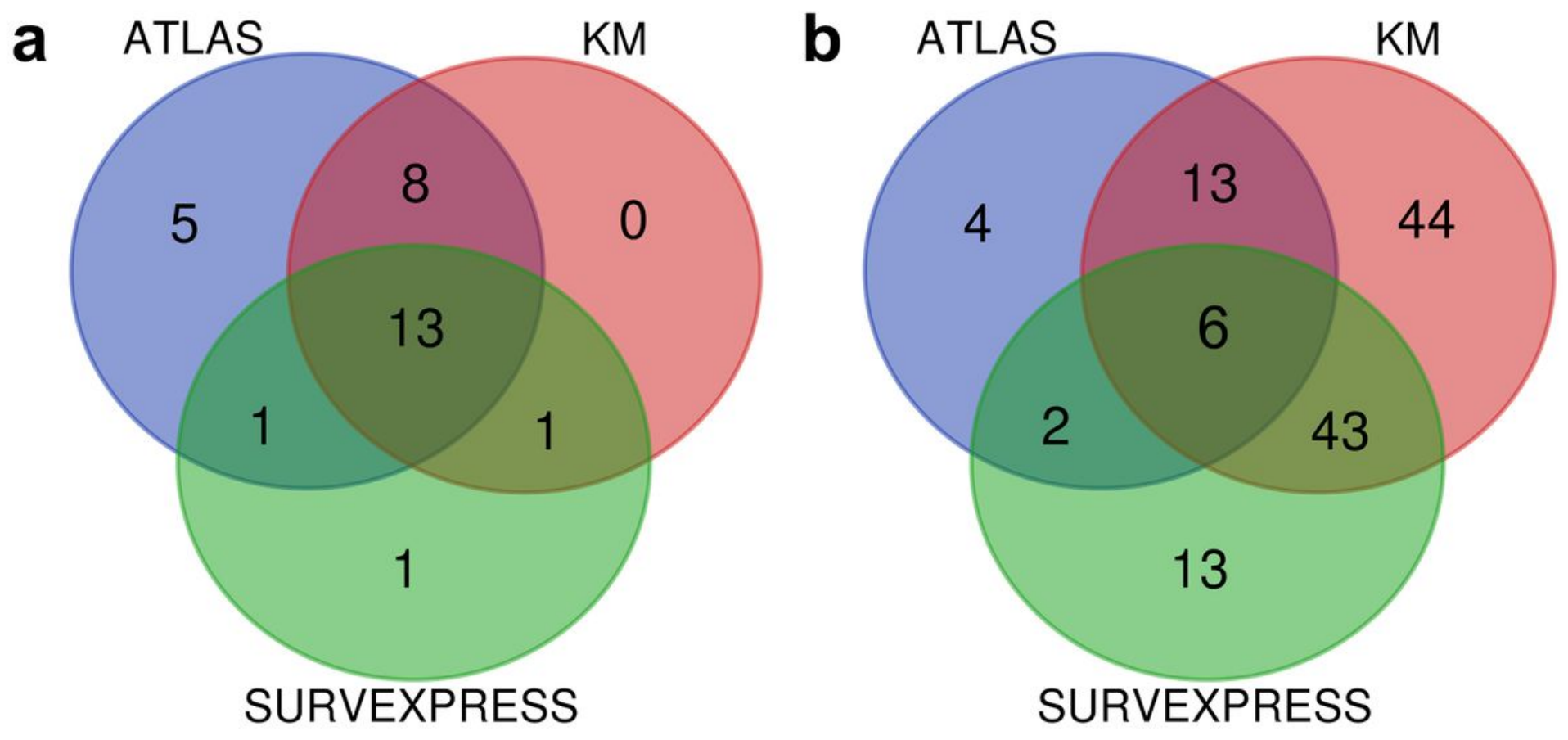

Figure 6 
Venn diagram of the overlapping parts of the three platforms, including the harmful and protective factors. There were 13 protective (a) and 6 harmful factors (b) in these five datasets.
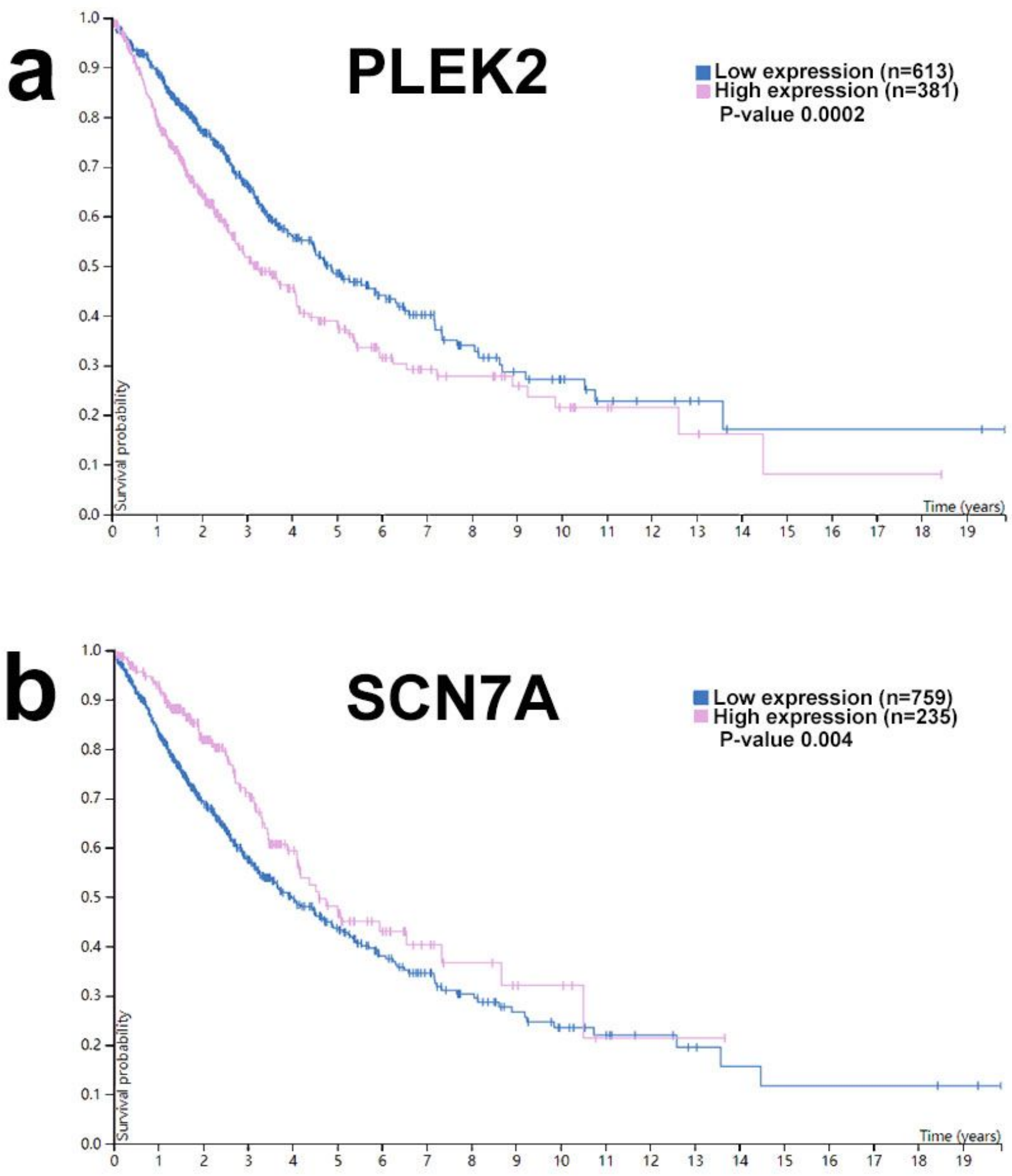

Figure 7

Overall survival curves based on pleckstrin 2 (PLEK2) and sodium voltage-gated channel alpha subunit 7 (SCN7A) expression. The survival curves of the high PLEK2 expression and low PLEK2 expression groups. (a) High PLEK2 expression was associated with poor overall survival (5-year survival, 39\% vs. 
$48 \%, \mathrm{P}<0.05$ ). (b) The survival curves of the high SCN7A expression and low SCN7A expression groups. Low SCN7A expression was associated with poor overall survival (5-year survival, $47 \%$ vs. $43 \%, \mathrm{P}<0.05$ ).

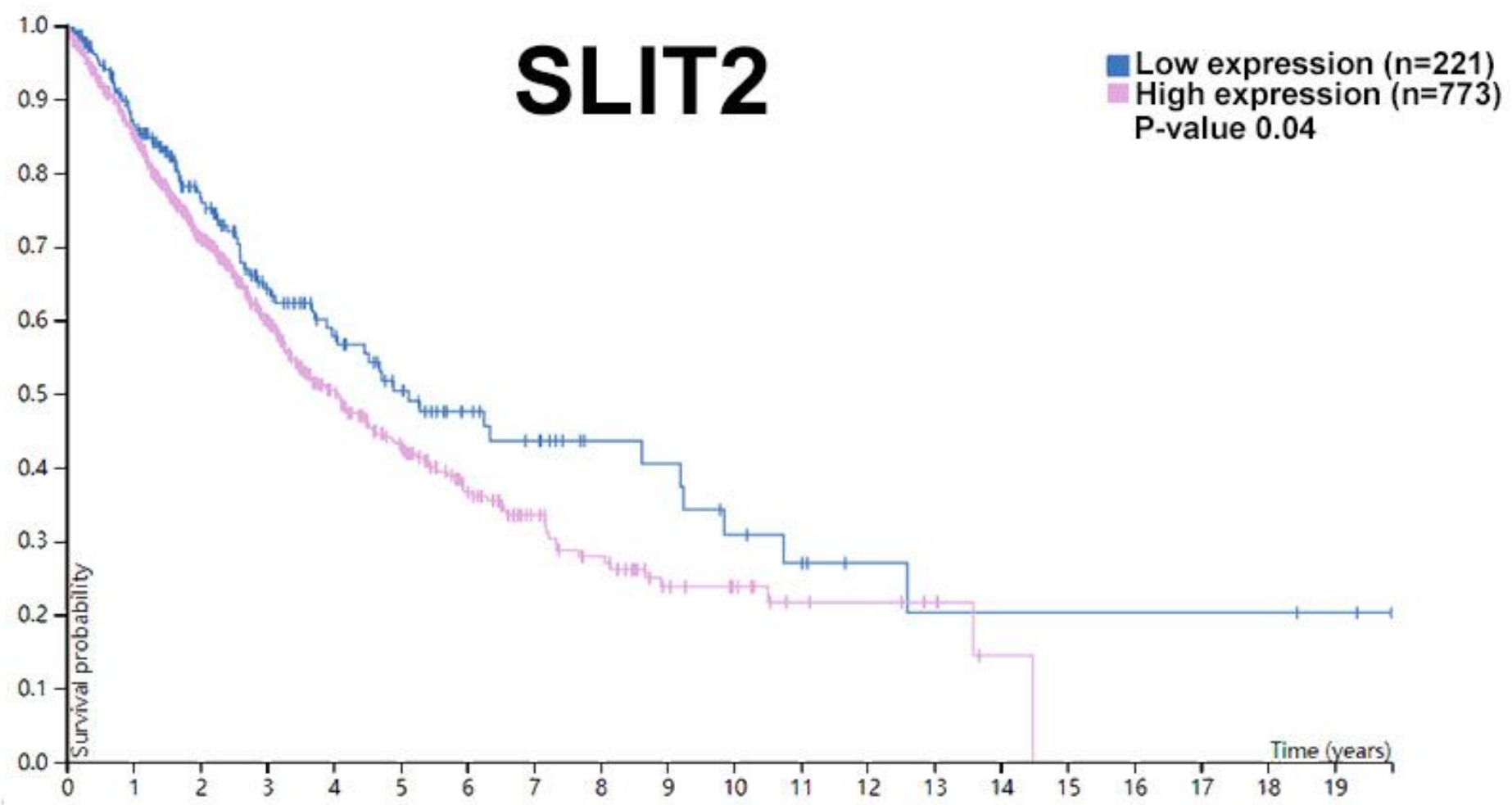

\section{Figure 8}

Overall survival curves based on SLIT2 expression. High SLIT2 expression was associated with poor overall survival (5-year survival, $43 \%$ vs. $50 \%, \mathrm{P}<0.05)$. 